\title{
Magnetic stimulation of the cerebellum could restore swallowing
}

Stimulating the cerebellum with transcranial magnetic stimulation (TMS) induces a motor response in the pharynx that could be used to treat swallowing disorders, according to researchers from the UK. "We found that direct stimulation of the cerebellum does evoke responses in the pharynx," explains Shaheen Hamdy, lead investigator, from the University of Manchester.

TMS is a noninvasive method for stimulating areas of the brain using short pulses of high-intensity magnetic fields. The researchers have already used this technique to show cortical control over swallowing, and wanted to investigate the influence of the cerebellum on pharyngeal motor activity. "Virtually nothing is known about how the human cerebellum controls swallowing," says Hamdy.

Equal numbers of healthy men and women $(n=16)$ aged between 19 years and 57 years underwent TMS. Electromyographic traces-showing the electrical activity of the pharyngeal muscles-were recorded by electrodes
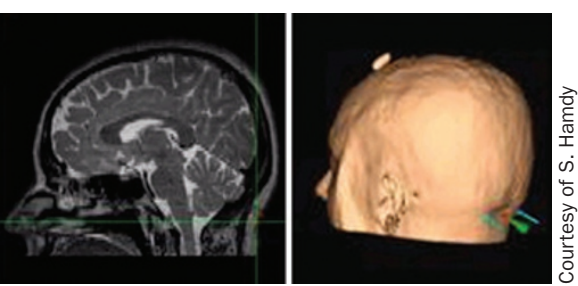

in an intraluminal catheter positioned in the pharynx. TMS was delivered through magnetic coils placed tangentially to the scalp during stimulatory pulses, and held at a right angle with only one edge in contact with the scalp for a sham (control) pulse. All participants received both stimulatory and sham pulses, and the pharyngeal motor activity after each was compared. No adverse events occurred after TMS.

The pulses were applied to the cerebellum-over the midline and both hemispheres - and the cortex to measure the effect of stimulating different areas of the brain on the amplitude of signals in the pharynx and the time between stimulation and motor activity (response latency).
TMS of the cerebellum alone evoked pharyngeal motor responses, indicating a direct signaling pathway between the cerebellum and pharynx. However, when the cerebellum was conditioned with TMS prior to cortical stimulation, the amplitude of the pharyngeal response strongly increased, and a single pulse could excite the cortical swallowing pathway for up to $200 \mathrm{~ms}$.

The investigators are now exploring whether prolonged stimulation of the cerebellum can excite the pharyngeal motor responses for seconds, minutes-or even hours-so that TMS could be used as a noninvasive therapy after brain injury. "If this technique can be used to enhance swallowing, it could be adapted to treat dysphagic disorders," concludes Hamdy.

Andy McLarnon

Original article Jayasekeran, V. et al. Non-invasive magnetic stimulation of the human cerebellum facilitates cortico-bulbar projections in the swallowing motor system. Neurogastroenterol. Motil. doi:10.1111/ j.1365-2982.2011.01747.x 